\title{
Correlation between Communication Skills and Nurses' Standardized Communication in Hazrat Rasoul Akram Complex, 2014
}

\author{
Behnaz Kermani ${ }^{1,2}$, Hasan Darvish ${ }^{2 *}$, Seyed Ali Akbar Ahmadi², Akram Bani Asadi ${ }^{3}$ Pirhossein Kolivand $^{4}$ \\ ${ }^{1}$ Office of Vice-Chancellor for Management and Resources Development, Iran University of Medical Sciences, Tehran, Iran \\ ${ }^{2}$ Department of Public Administration, Payame Noor University, Tehran, Iran \\ ${ }^{3}$ Department of Health Management and Economics, School of Public Health, Tehran University of Medical Sciences, Tehran, Iran \\ ${ }^{4}$ Shefa Neuroscience Research Center, Khatam Alanbia Hospital, Tehran, Iran
}

\section{A BSTRACT}

Introduction: The ability to communicate is crucial for the people working in the health system, particularly the nurses. Communication barriers between the medical team disrupt teamwork and resulting in a lower safety and quality of care. The aim of this study was to investigate the correlation between nurses' communication skills and standardized communication. Materials and Methods: This is a descriptive study and was conducted in the 2014. The study population was the nursing staff of Hazrat Rasoul Akram Complex. Eligible nurses were selected randomly. Two questionnaires were used to collect information on interpersonal communication skills and the standard situation, background, assessment and recommendation (SBAR) communication tool and demographic data. Results: Less than $45 \%$ of nurses had good communication skills and less than $50 \%$ of nurses were used standards communication items. There was a significant correlation between interpersonal skills and communication standards as well as individual skills with situation, background, assessment, and recommendation. Conclusion: Improvement of communication skills and standardized communication increase coordination between the medical team and providing information about the background and situation of the patient. Moreover, reduction of the likelihood of errors and ignoring information improve the efficiency, reduce dissatisfaction, and provide optimum care.

\section{Key words:}

1. Communication

2. Clinical Competence

3. Reference Standards

4. Nurses

5. Hospitals

*Corresponding Author: Hasan Darvish

E-mail:dr.darvish43@gmail.com 
همبستگى بين مههارتهاى ارتباطى و ارتباط استاندارد يرستاران در مجتمع حضرت رسول اكرم، بوسا

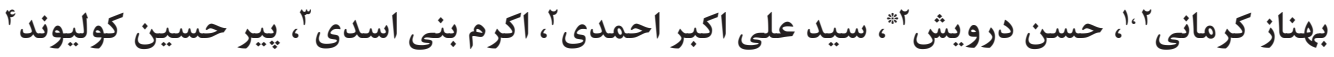

$$
\begin{aligned}
& \text { 'دفتر معاونت توسعه مديريت و منابع، دانشكاه علوم يزشكى ايران، تهران، ايران } \\
& \text { rكروه مديريت دولتى، دانشخاه يِيام نور، تهران، ايران }
\end{aligned}
$$

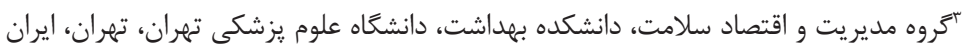

$$
\begin{aligned}
& \text { †مركز تحقيقات علوم اعصاب شفا، بيمارستان خاتمالانبياء، تهران، ايران }
\end{aligned}
$$

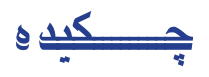

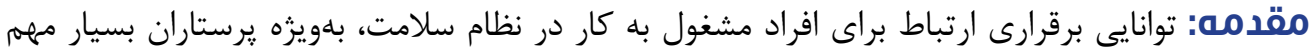

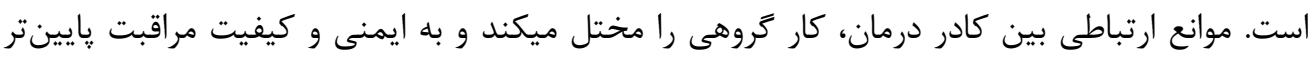

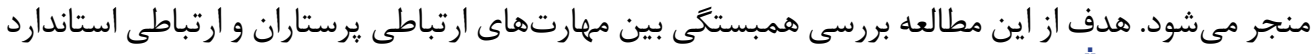

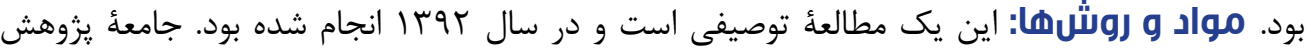

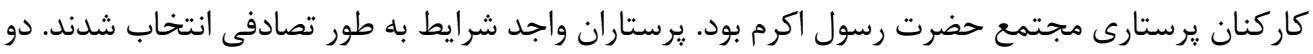

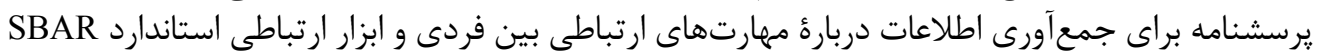

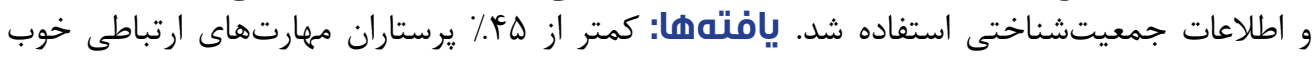

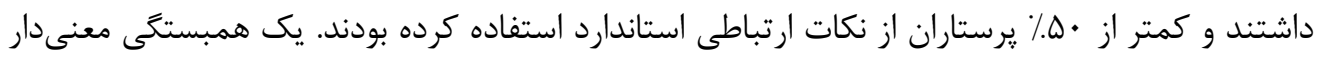

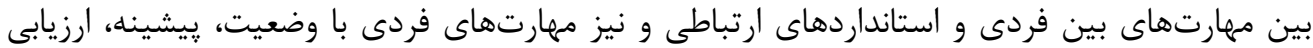

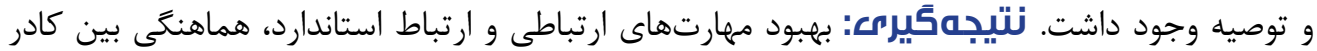

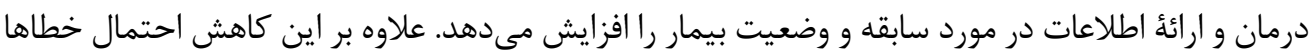

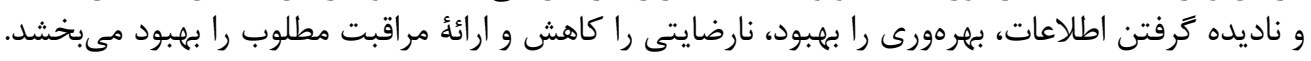

r

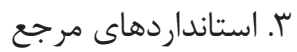

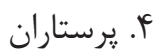

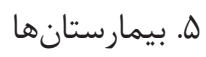

"نويسنده مسئول: حسن درويش آدرس الكترونيكى: dr.darvish43@gmail.com 


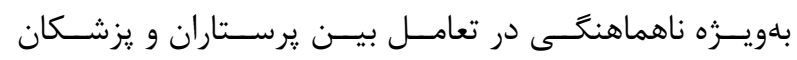

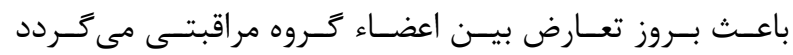

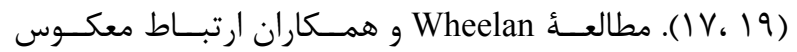

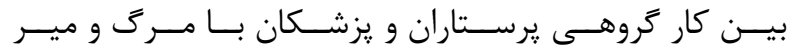

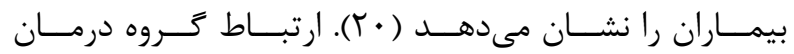

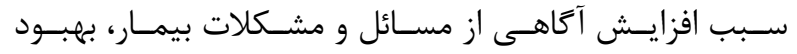

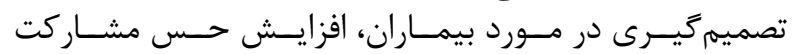

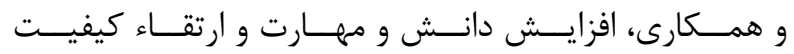

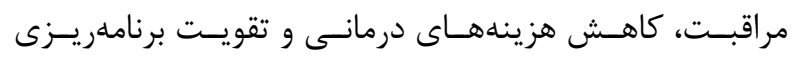

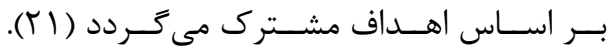

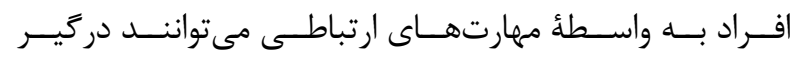

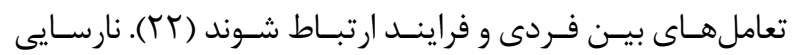

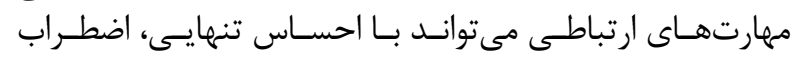

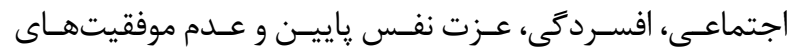

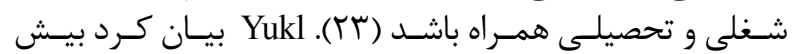

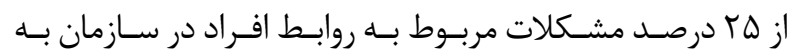

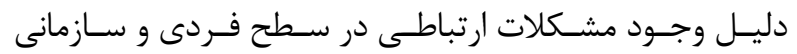

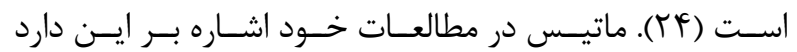

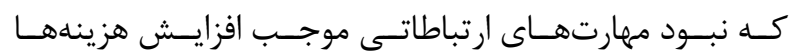

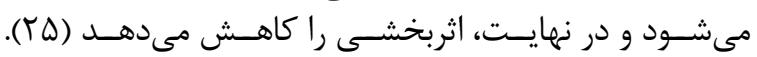

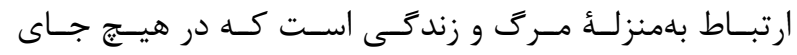

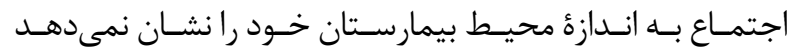

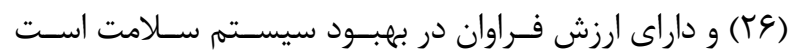

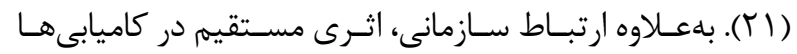

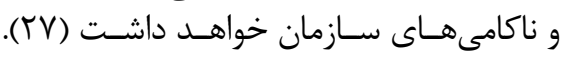

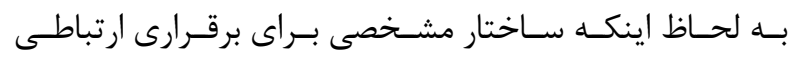

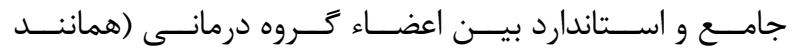

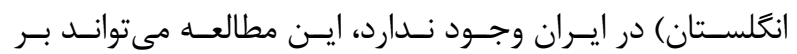

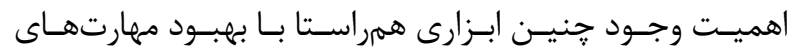

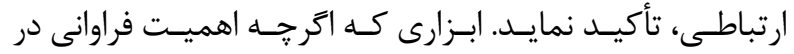

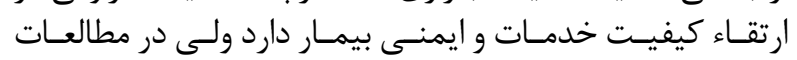

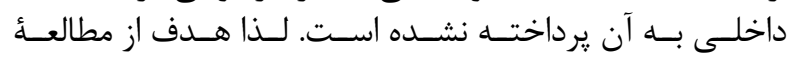

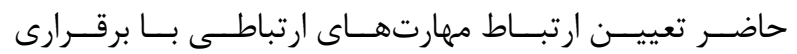

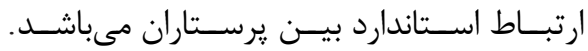

مواد و روشها - - مان

ايسـن مطالعـه از نــوع توصيفـى بــوده كـهـ در زمسـتان سـال

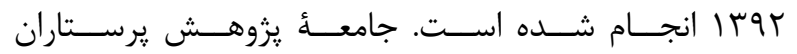

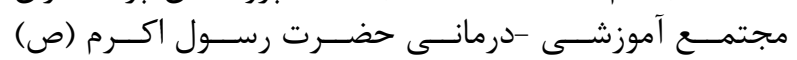

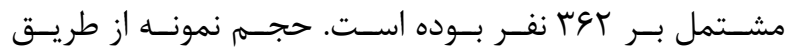
فرمـول نمونه

$(\mathrm{p}, \mathrm{q}=\cdot / \mathrm{Q}$ ! $Z=) / 94$ ؛

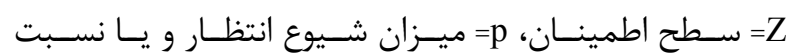

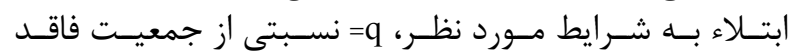

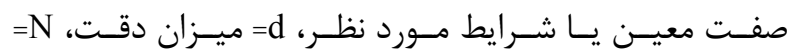
حجسم جامعسه معند

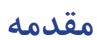

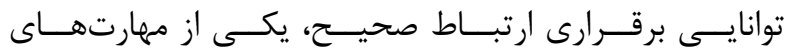

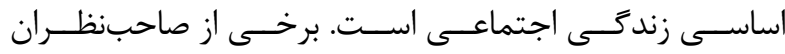

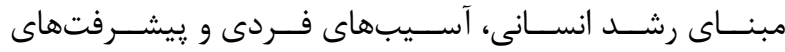

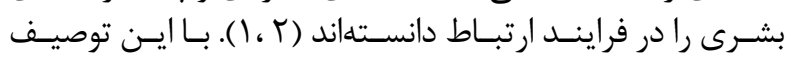

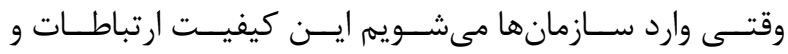

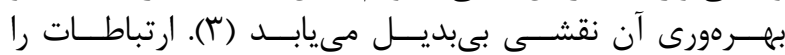

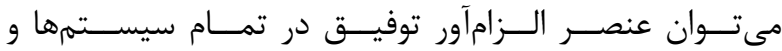

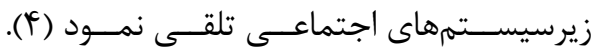

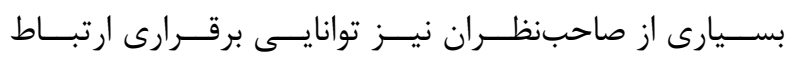

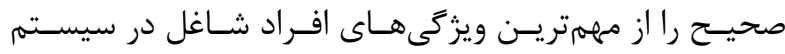

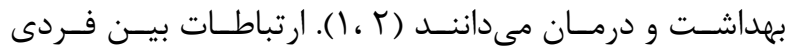

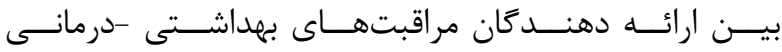

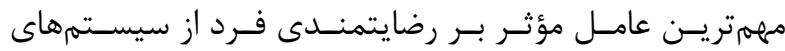

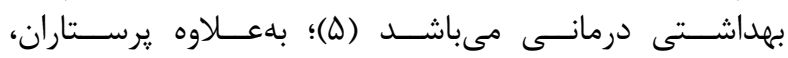

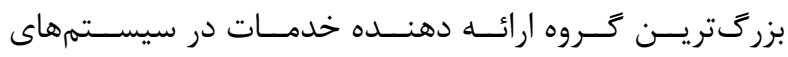

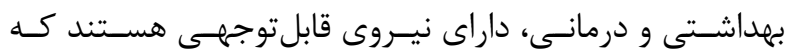

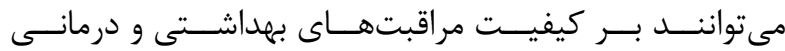

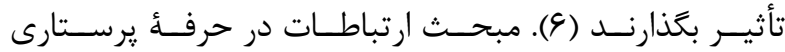

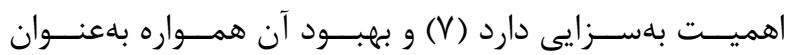

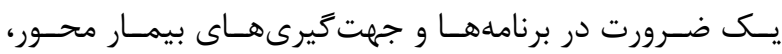

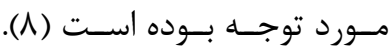

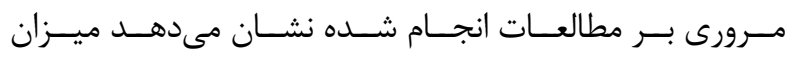

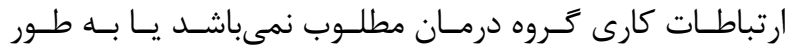

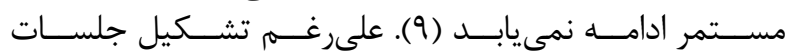

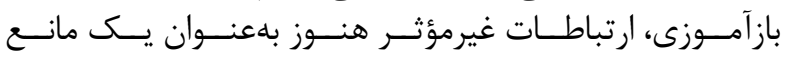

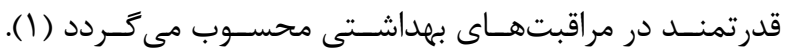

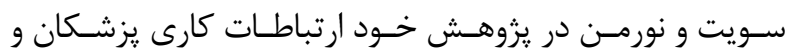

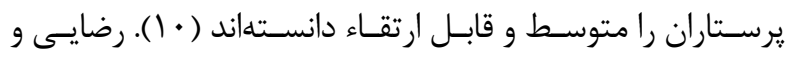

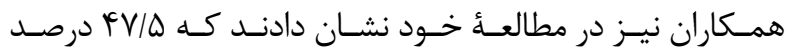

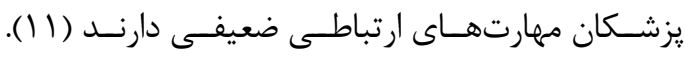

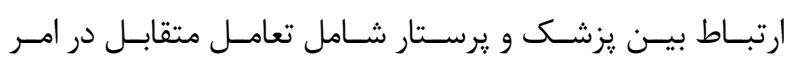

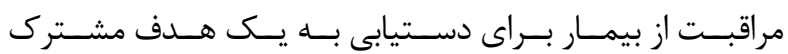

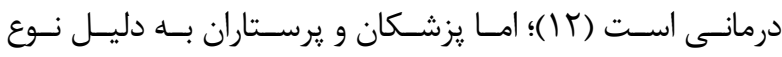

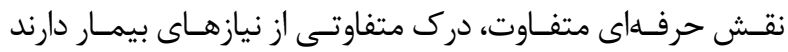

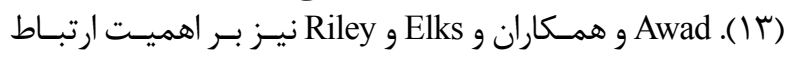

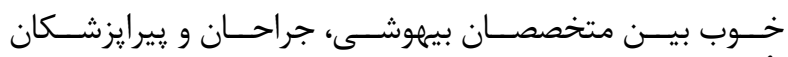

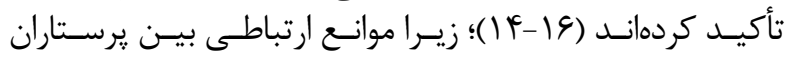

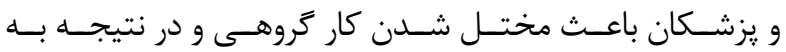

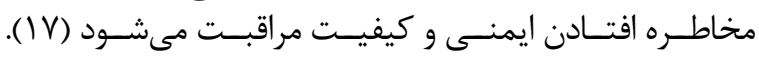

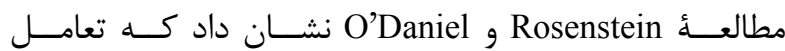

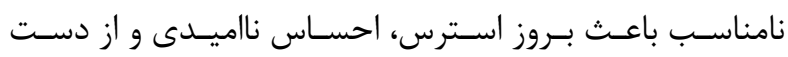

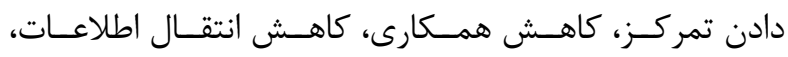

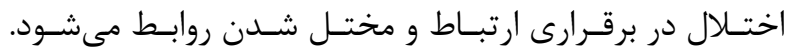

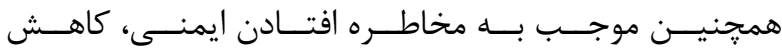

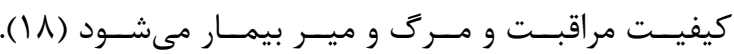




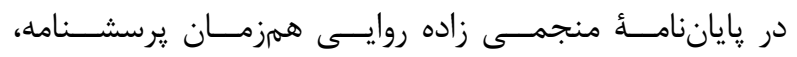

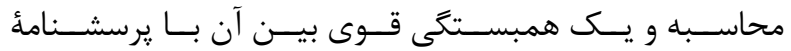

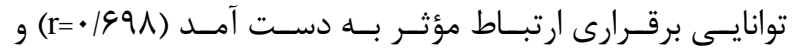

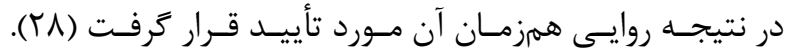

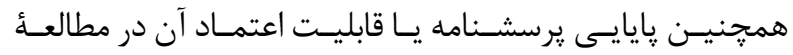

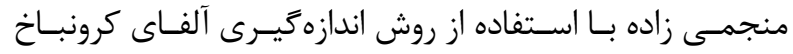

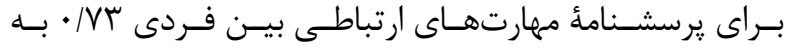

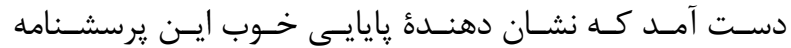

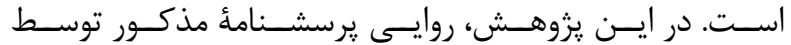

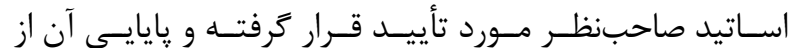

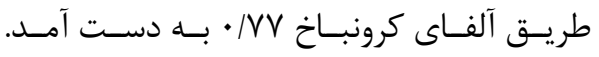

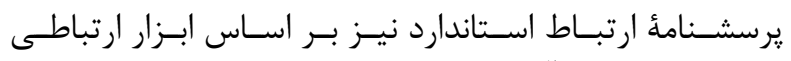

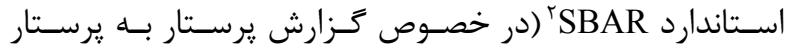

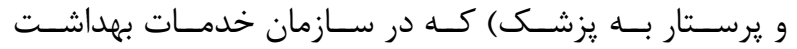

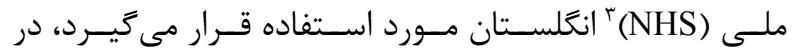

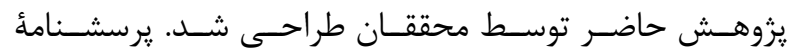

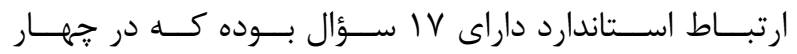

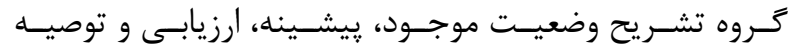

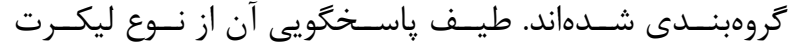

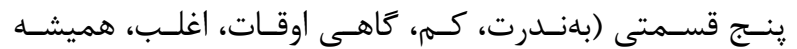

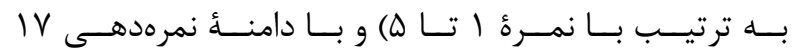

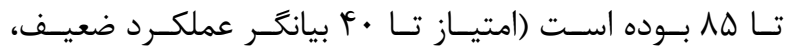
بره

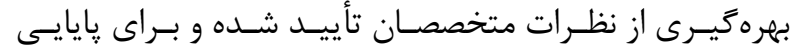

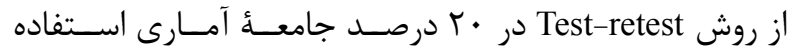

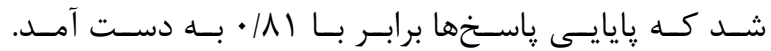

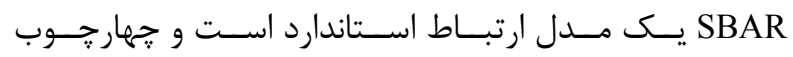

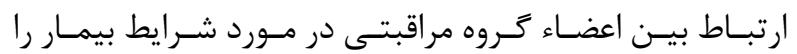

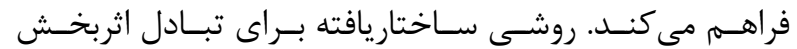

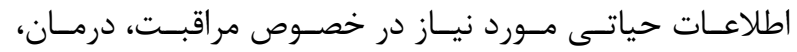

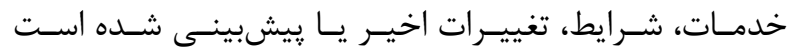

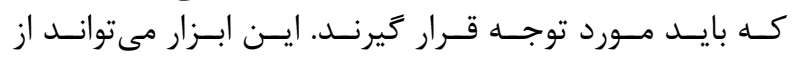

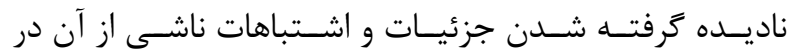

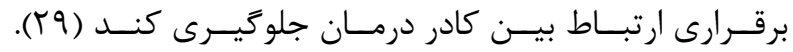

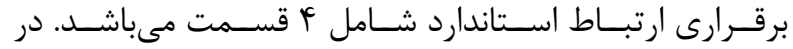

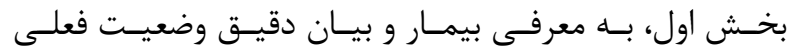

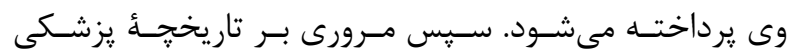

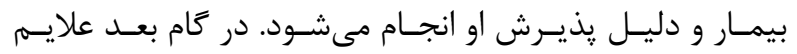

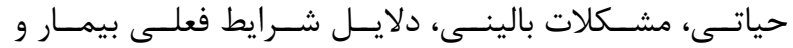

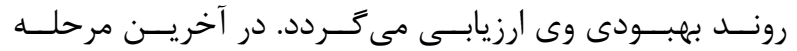

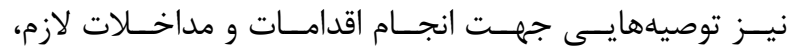

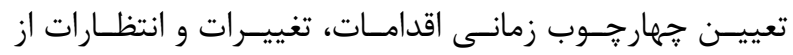

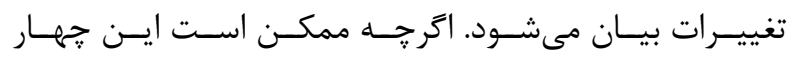

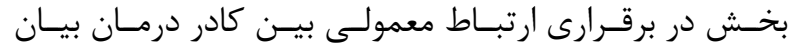

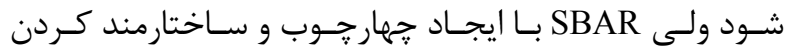

$$
n=\frac{N Z^{r} p q}{(N-1) d^{r}+Z^{r} p q}
$$

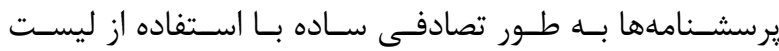

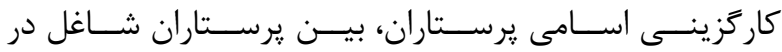

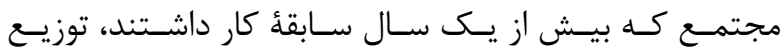

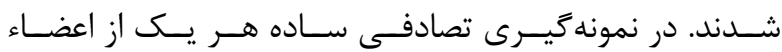

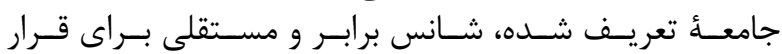

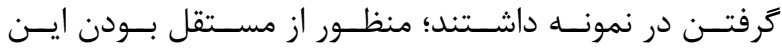

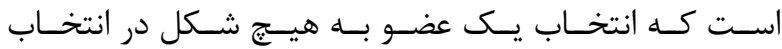

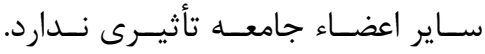

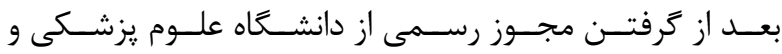

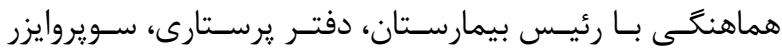

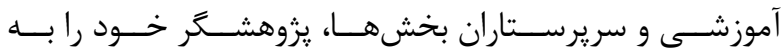

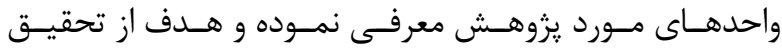

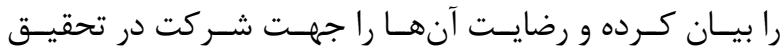

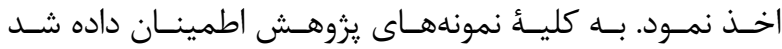

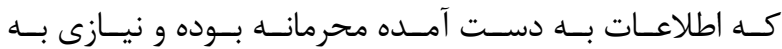

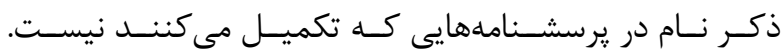

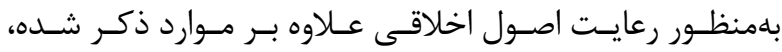

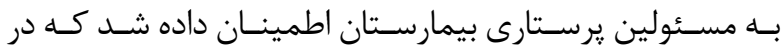

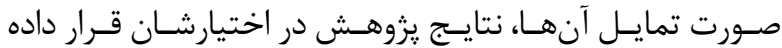

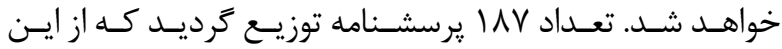

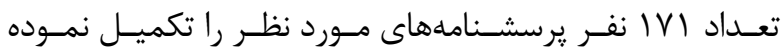

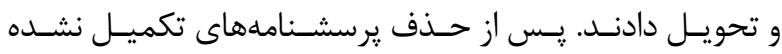

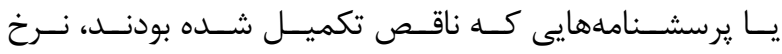

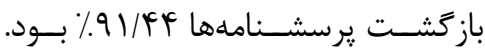

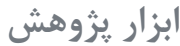

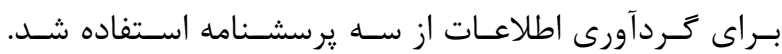

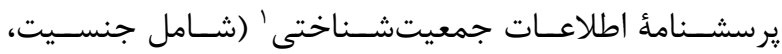

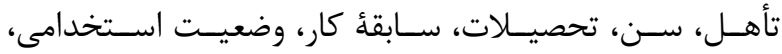

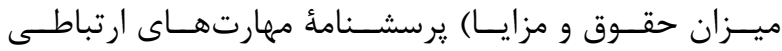

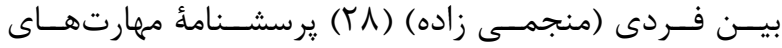

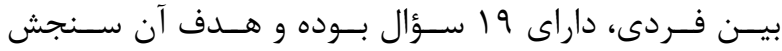

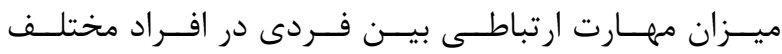

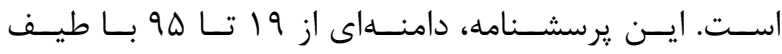

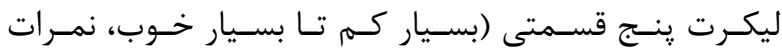

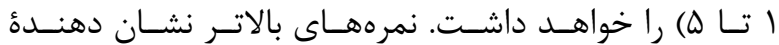

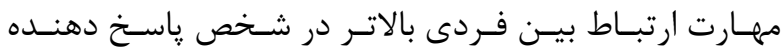

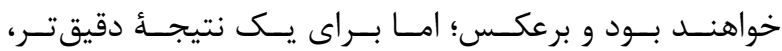

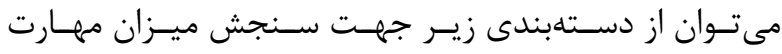

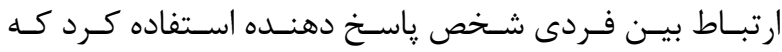

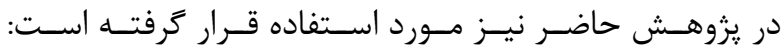

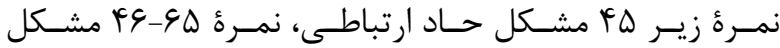

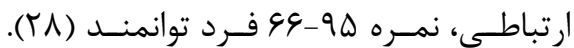




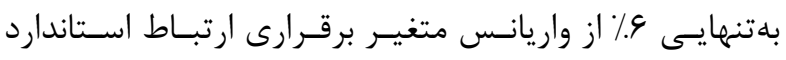

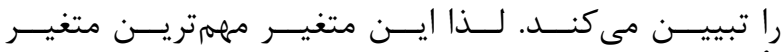

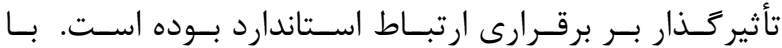

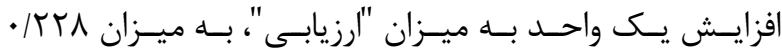

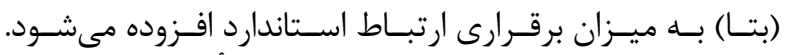

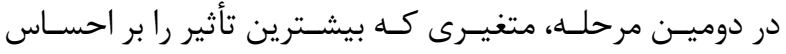

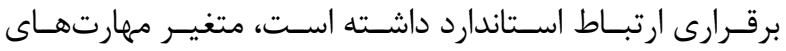

فـردى بـوده اسـت.

جدول ا- مشخصات جمعيتشناختى شركت كنندكان در مطالعه.

\begin{tabular}{|c|c|c|c|}
\hline ثرحد & تعداث & مشخصات & \\
\hline १४/VF & 194 & زن & \multirow{2}{*}{ جنسيت } \\
\hline$\Delta / T G$ & 9 & ل مرد & \\
\hline$\Delta \cdot / \lambda \Lambda$ & $A V$ & هجرد & \multirow{2}{*}{ وضعيت تأهل } \\
\hline$F q / 1 r$ & Af & متأهل & \\
\hline سזب/קו & 11. & $r \cdot-r$. & \multirow{3}{*}{ سن (سال) } \\
\hline$r r / r r$ & $\Delta V$ & ril- & \\
\hline$r / T F$ & $f$ & $+1-0+$ & \\
\hline$\Delta T / \cdot F$ & IfF & لبسيانس & \multirow{3}{*}{ ميزان تحصيلات } \\
\hline $1 \Delta / \gamma 9$ & rV & فوق لبسأنس & \\
\hline W/V & $r$ & 25: & \\
\hline$\Delta+/ \lambda \Lambda$ & AV & $1-Y$ & \multirow{4}{*}{ سابقة كار (سال) } \\
\hline$r \% / 0$ & $\Delta q$ & $1-1 \%$ & \\
\hline N/9 & if & $|\Delta-r|$ & \\
\hline STH & 11 & $r r-r$. & \\
\hline $\mathrm{V} / \mathrm{T}$ & ir & طرحى & \multirow{5}{*}{ وخعيت استخدامي } \\
\hline sR/OV & $1 \cdot V$ & ربسمى & \\
\hline$\varphi / 9 \lambda$ & $\wedge$ & رسمى [ززعايشى & \\
\hline$\varphi / 9 \lambda$ & $\wedge$ & بيبمانى & \\
\hline$r 1 / .0$ & re & قراردائى & \\
\hline$F 9 / \mathrm{YA}$ & $\lambda$. & دارد & \multirow{2}{*}{ شيفت } \\
\hline ST/T & 9) & تدارد & \\
\hline $18 / \mathrm{Yq}$ & TV & زير 1 & \multirow{4}{*}{ حقوق (به ميليون تومان) } \\
\hline GNFT & $11 \mathrm{~V}$ & $1-1 / 0$ & \\
\hline$Q / 9 P$ & iv & $1 / \Delta-Y$ & \\
\hline$\Delta / A \Delta$ & 1. & $r-r / \Delta$ & \\
\hline $11 / \mathrm{V}$ & Y. & سوبروايزر & \multirow{4}{*}{ روة شغلى } \\
\hline $19 / \mathrm{Al}$ & $r p$ & سريرسئار & \\
\hline y/1V & $r$ & الستف & \\
\hline SV/TO & 110 & بربستار & \\
\hline
\end{tabular}

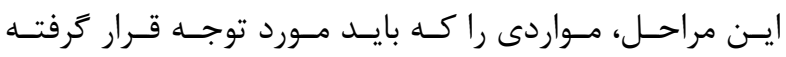

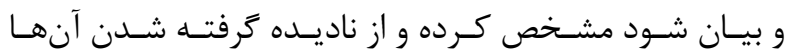

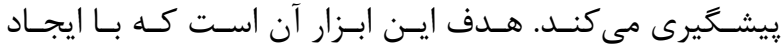

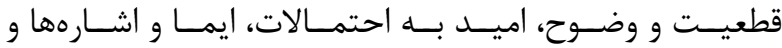

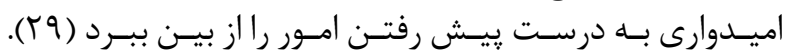

تجزيه و تحليل دادهها

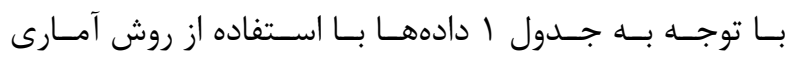

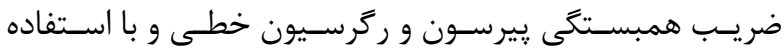

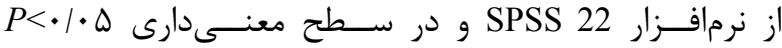

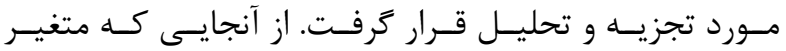

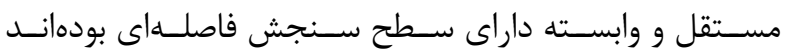

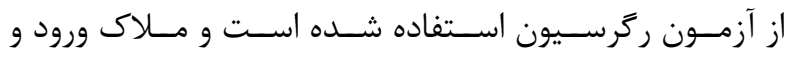

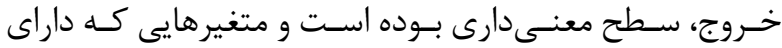

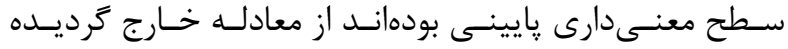

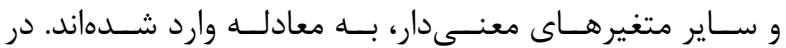

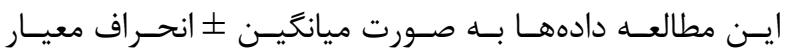

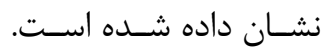

بافتهها

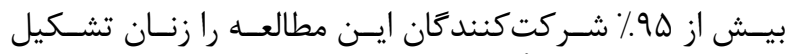

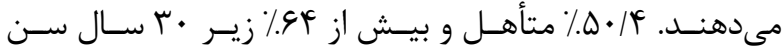

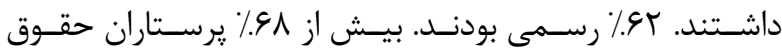

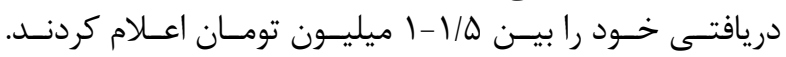

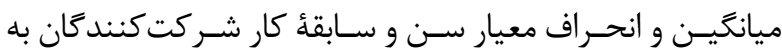

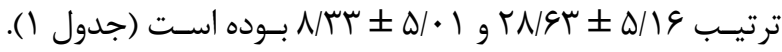

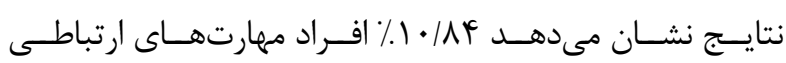

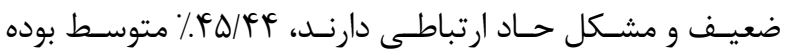

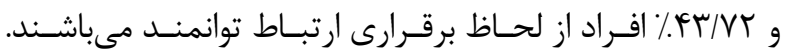

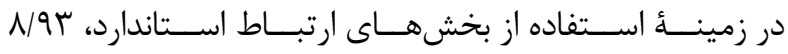

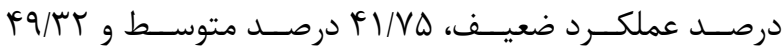

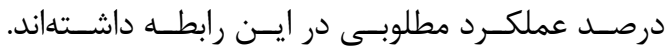

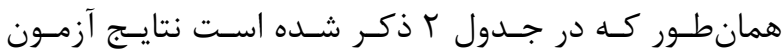

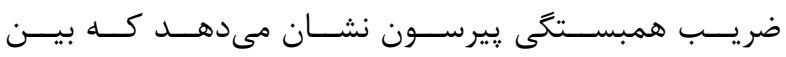

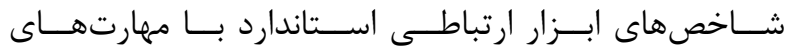

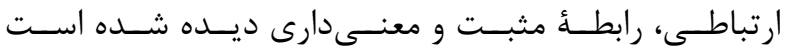

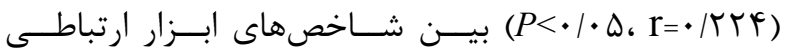

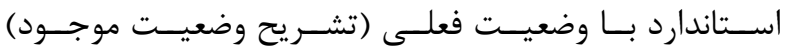

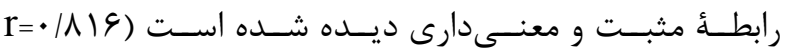

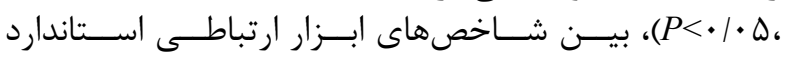

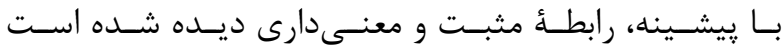
(P<•/•D، r=•/AFF)

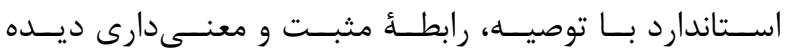

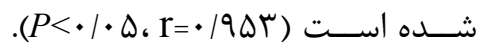

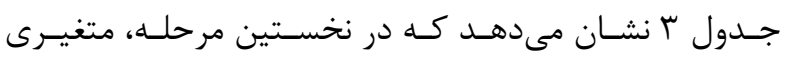

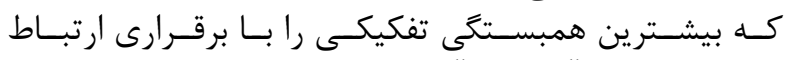

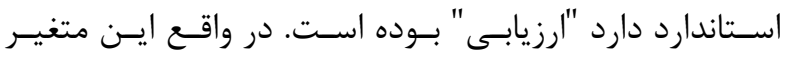




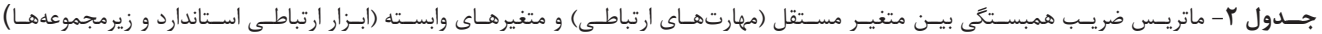

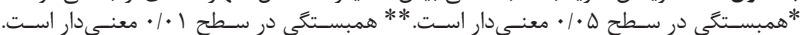

\begin{tabular}{|c|c|c|c|c|c|c|c|}
\hline البزار ارتباطى استاندارد & توصية & ارزيلبى & بيشينه & وضعيت فعلى تشبريح & هبارتهاى ارتباطى & & \\
\hline & & & & & 1 & r & \multirow{2}{*}{ مهارتهاي الرتباطى } \\
\hline & & & & & . & $P$ & \\
\hline & & & & 1 & $\left(^{*}\right) \cdot / 1 \mathrm{Vr}$ & $\mathbf{r}$ & \multirow{2}{*}{ وفيث فعلى -تشريج } \\
\hline & & & & & $.1+4 \Delta$ & $P$ & \\
\hline & & & 1 & $\left({ }^{* *}\right) \cdot / V A T$ & $\left(^{*}\right)$ - ror & $\mathbf{r}$ & \multirow{2}{*}{ ييشينه } \\
\hline & & & . & $+1 \cdots 1$ & $.1 \cdot 11$ & $P$ & \\
\hline & & 1 & $\left({ }^{* *}\right) \cdot$ / & $\left({ }^{* \pm}\right) \cdot / 9 \Gamma \lambda$ & $\left(^{*}\right) \cdot / r T \lambda$ & $\mathbf{r}$ & \multirow{2}{*}{ ارزيابي } \\
\hline & & . & $* 1 \cdots 1$ &.$/ \cdots 1$ & $\cdot 1 \cdot T r$ & $P$ & \\
\hline & 1 & $\left({ }^{* *}\right) \cdot / 9 Y \wedge$ & $\left({ }^{* *}\right) \cdot[\lambda \cdot F$ & $\left({ }^{* \star}\right) \cdot / \Delta \mathrm{VT}$ & $\left(^{*}\right) \cdot / / \vee q$ & $\mathbf{r}$ & \multirow{2}{*}{ توصيه } \\
\hline & . & $\cdot \cdots 1$ & $\cdot \cdots 1$ & 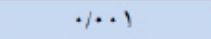 & $.1 \cdot F F$ & $P$ & \\
\hline 1 & $\left({ }^{* *}\right) \cdot /$ QT & $(* *) \cdot / 9 \Delta T$ & $\left({ }^{* *}\right) \cdot / 9 F F$ & $\left({ }^{* *}\right) \cdot / 1 / 8$ & $\left({ }^{*}\right) \cdot / Y T F$ & $\mathbf{r}$ & \multirow{2}{*}{ ابزار الرتباطى استاتداره } \\
\hline$\ddot{\theta}$ & $\cdot(\cdots)$ & 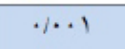 &. $\mid \cdots 1$ &.$/ \ldots 1$ & $.1 \cdot T \Delta$ & $P$ & \\
\hline
\end{tabular}

جدول ب- نتايج آزمون رَّر سيون خطى مؤلفههاى مؤثر بر ارتباط استاندارد.

\begin{tabular}{|c|c|c|c|c|}
\hline $\mathrm{R}$ & سطح معنى دارى & $\mathrm{T}$ & 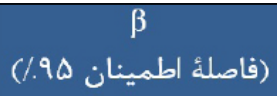 & \\
\hline - / TAT & $\cdot / \cdot 1$ & $r / T_{1}$ & - /tr人 & ارزيابى \\
\hline$\cdot|\Delta| \cdot$ & $\cdot \mid \cdots \cdot 1$ & $T / T V$ & - /TtK & مرارتهاى ارتباطى فردى \\
\hline - NTY & $\cdot / \cdot 1$ & $V / P \Lambda$ & $\cdot / 1 \vee 9$ & توصيه \\
\hline$\cdot / 911$ & $\cdot / \cdot F \Delta$ & $q / V F$ & $\cdot / 1 \mathrm{VT}$ & وضعيت فعلى (تشريح وضعيت موجود) \\
\hline
\end{tabular}

بحث و نتيجه

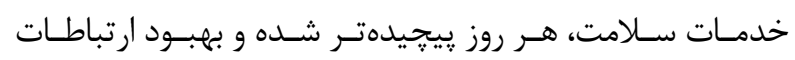

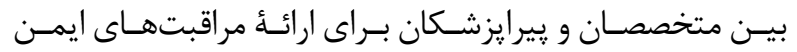

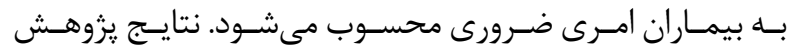

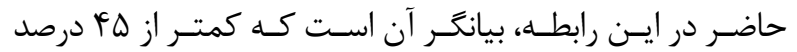

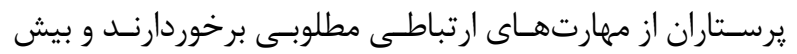

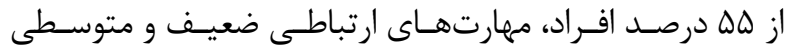

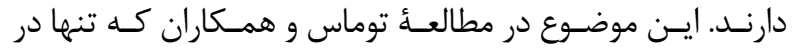

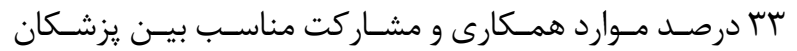

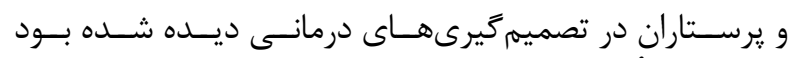

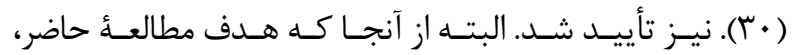

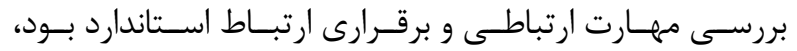

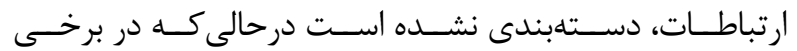

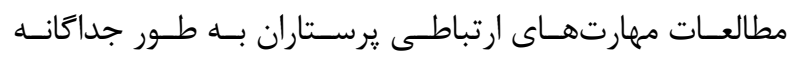

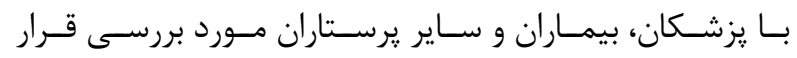

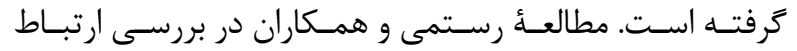

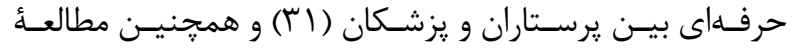

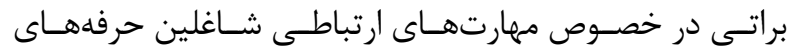

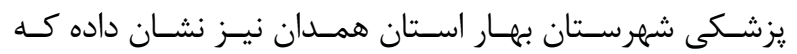

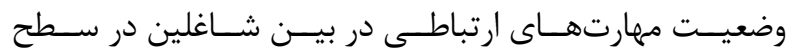

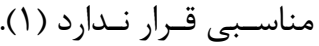

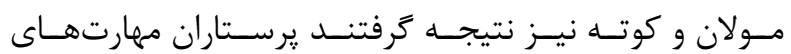

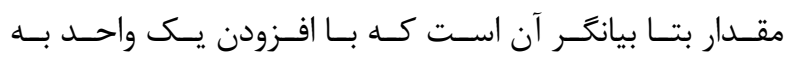

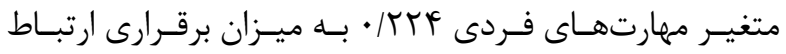

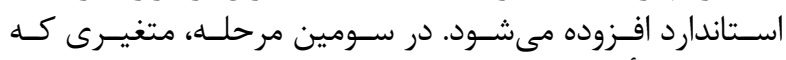

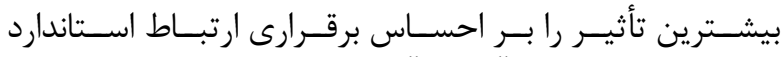

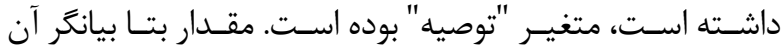

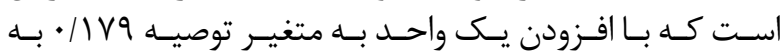

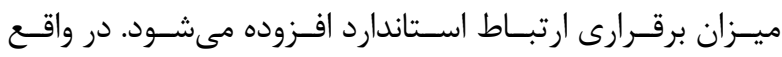

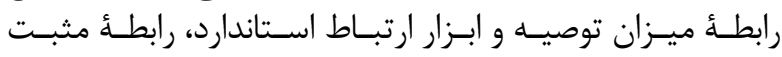

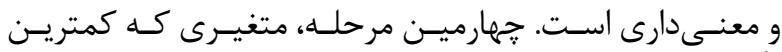

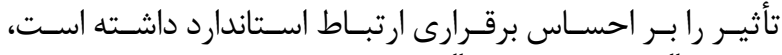

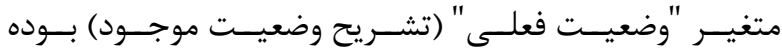

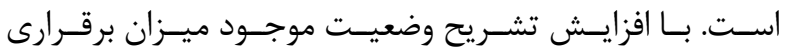

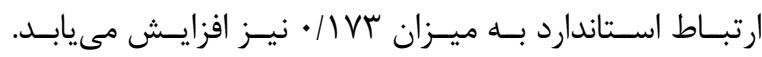

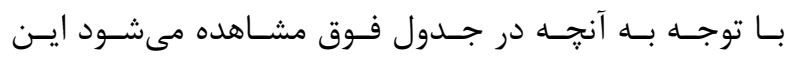

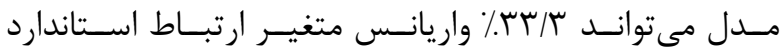

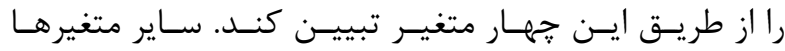

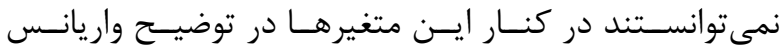

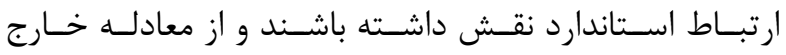

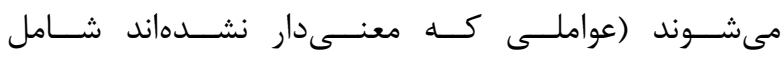

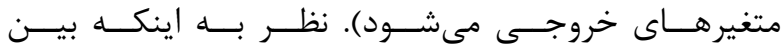

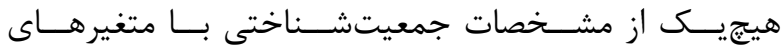

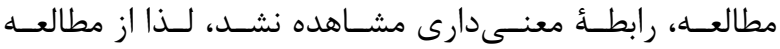

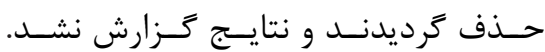




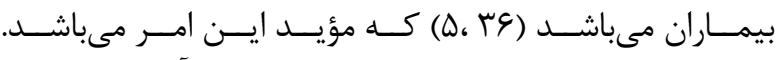

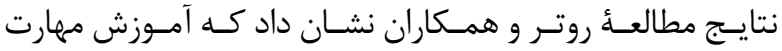

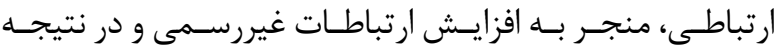

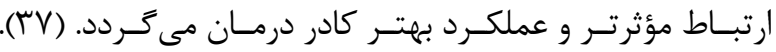

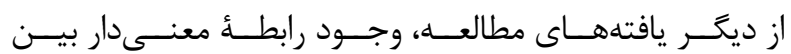

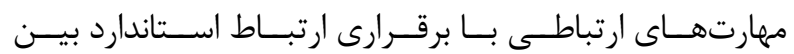

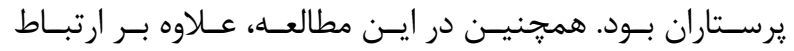

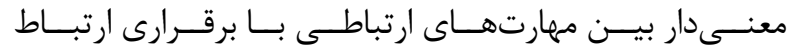

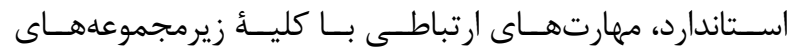

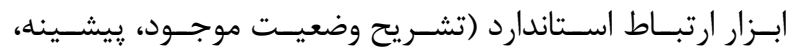

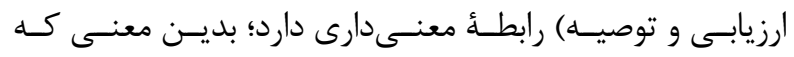

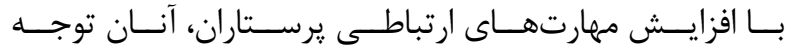

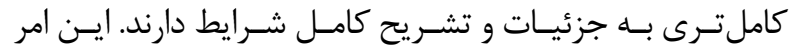

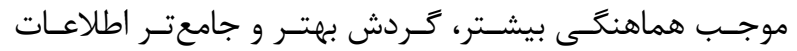

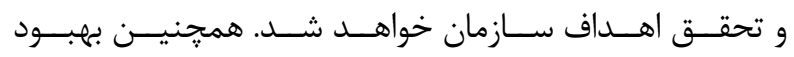

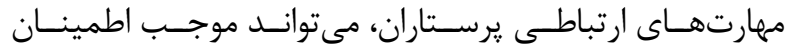

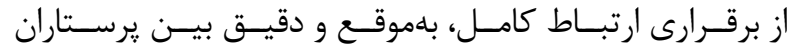

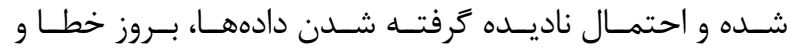

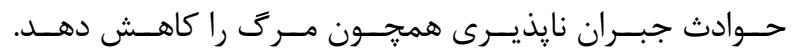

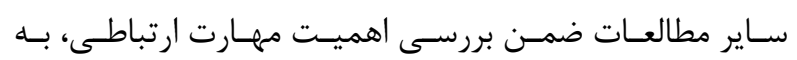

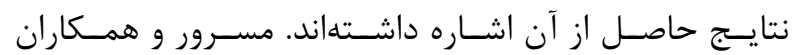

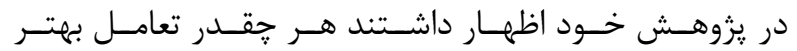

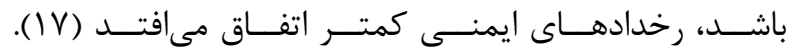

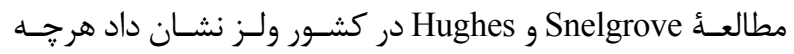

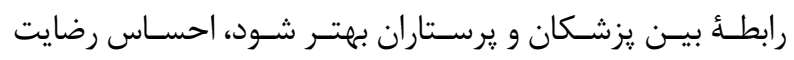

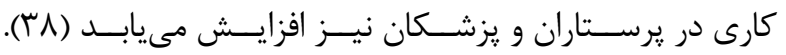

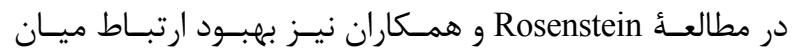

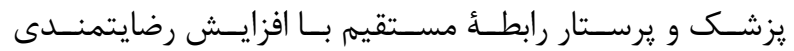

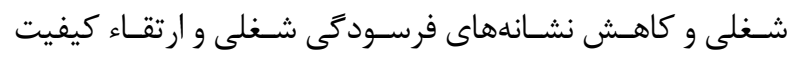

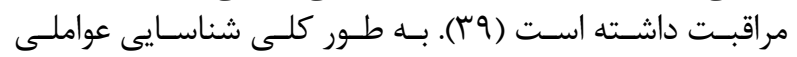

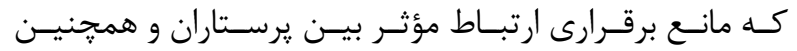

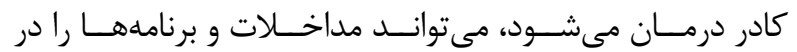

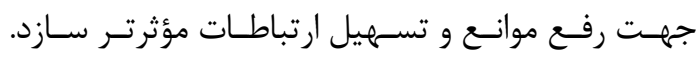

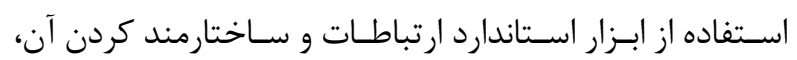

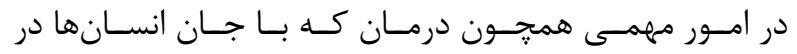

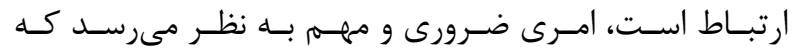

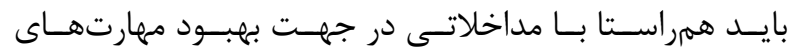

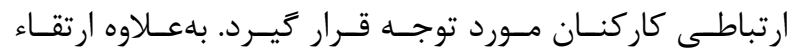

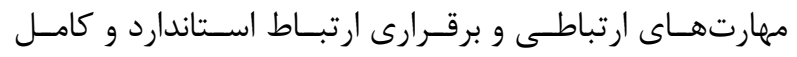

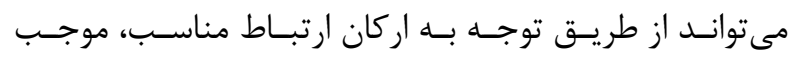

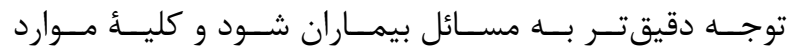

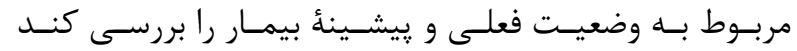

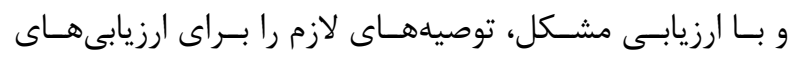

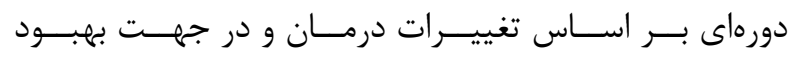
وضعيـت بيمـار بيـان كنـد.

توانايسى كادر درمــان در كار كــردن بــا يكديخـــر بهعنــوان

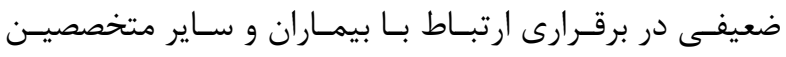

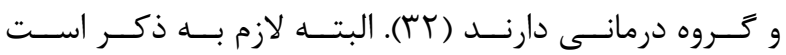

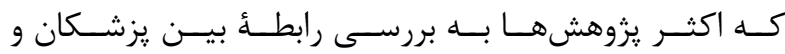

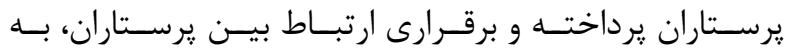

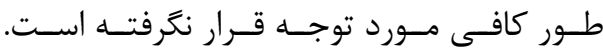

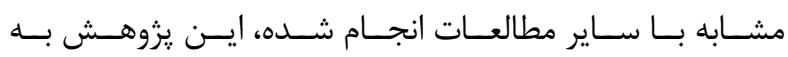

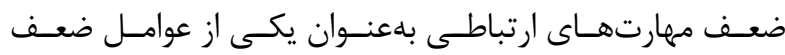

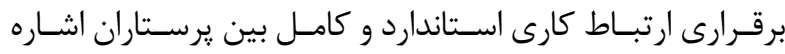

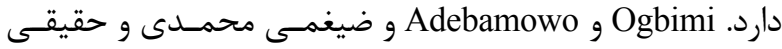

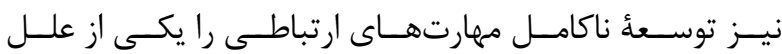

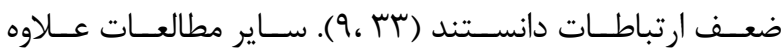

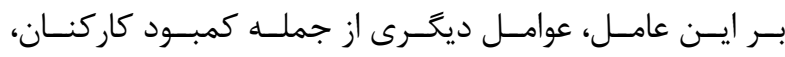

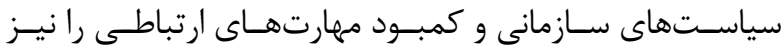

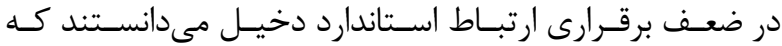

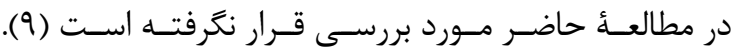

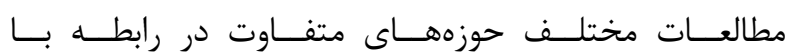

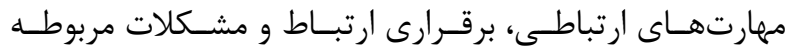

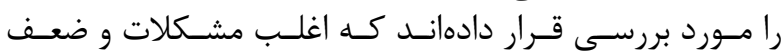

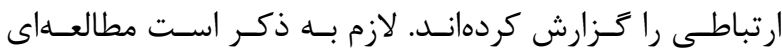

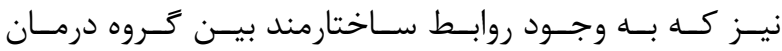

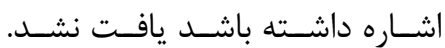

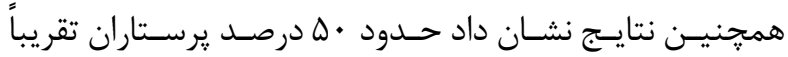

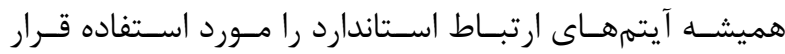

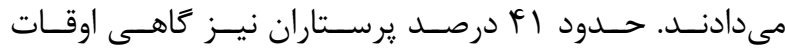

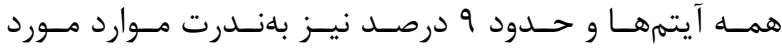

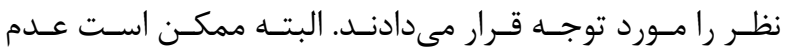

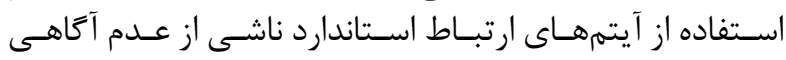

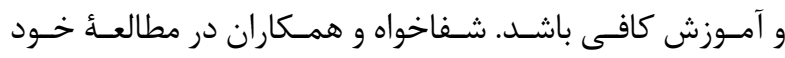

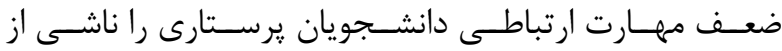

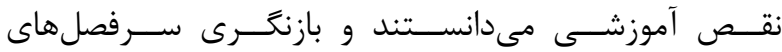

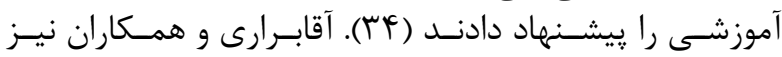

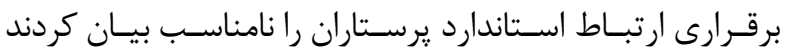

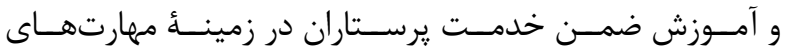

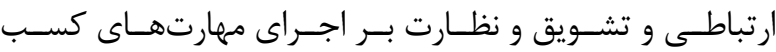

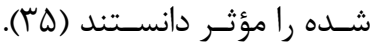

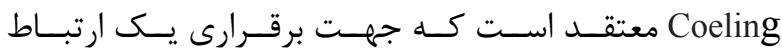

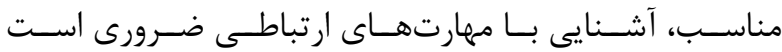

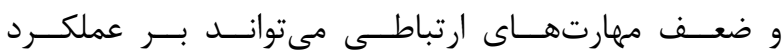

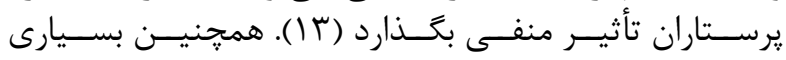

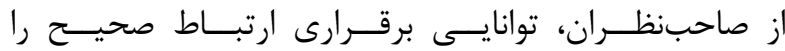

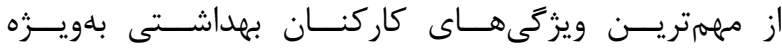

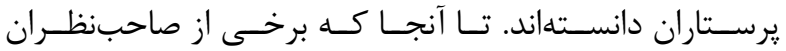

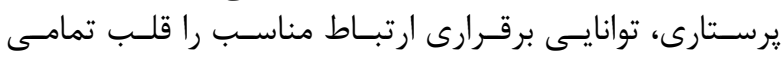

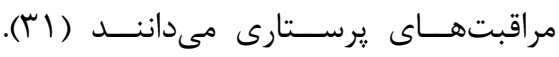

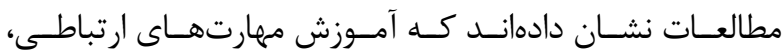

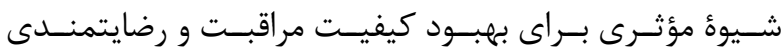




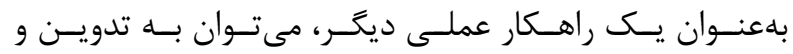

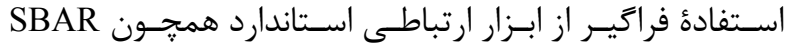

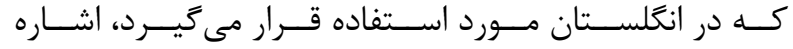

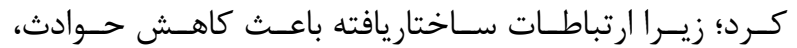

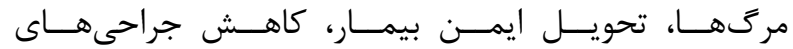

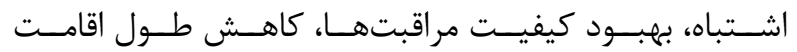

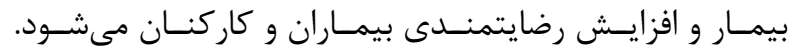

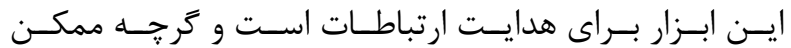

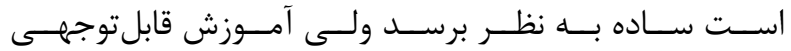

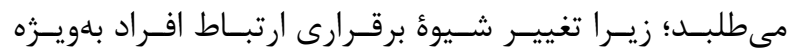

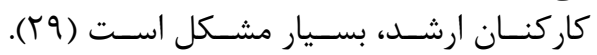

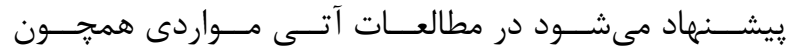

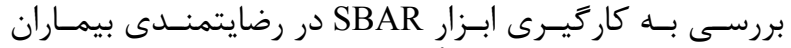

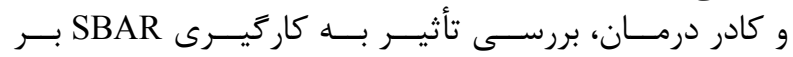

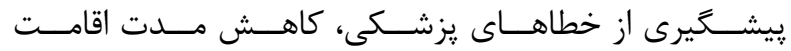

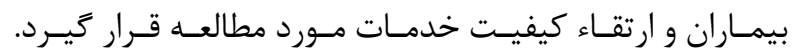

$$
\text { تشكر و قدردانى }
$$

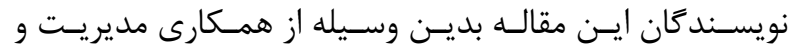

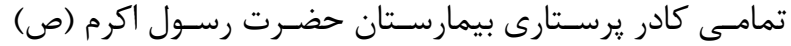

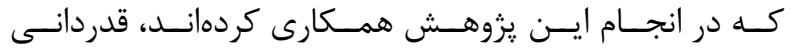

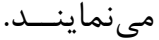

1. Barati M, Afsar A, Ahmadpanah M. Professional communication skills of medical practitioners in Bahar city in 2010. J Hamadan Univ Med Sci. 2012; 19(1): 62-9.

2. Barati M, Moeini B, Samavati E, Salehi OM. Assess medical students' communication skills: verbal skills, listening and feedback. Journal of School of Nursing and Midwifery of Urmia. 2012; 10(2): 145-54.

3. Zarei Matin H, Yusef Zadeh S. Explaining the parameters of the communication skills of managers and its role in job satisfaction. Journal of Educational Psychology, Islamic Azad University, Tonekabon Branch. 2010; 1(2): 29-48.

4. Tabibi SJ, NassiriPour AA, Zahiri Abyaneh Z. Relationship between communication skills of managers and employees motivatation in Modarres hospital in Tehran. Hospital Quarterly. 2013; 12(2): 73-80.

5. Farmahin Farahani M, Kashaninia Z, Hosseini MA, Biglarian A. The impact of communication skills training on patients' satisfaction with nursing communication. Iranian Journal of Nursing Research. 2007; 1(3): 47-54.

6. Nasiri Poor A, Saeedzadeh Z, Sabahi Bigdeli M. Correlation between nurses' communication skills and Quality of inpatient services in hospitals of Kashan

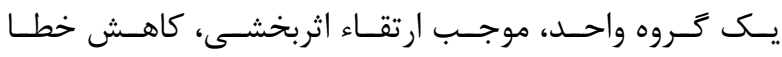

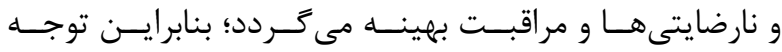

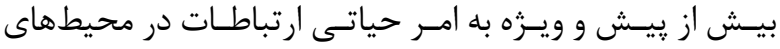

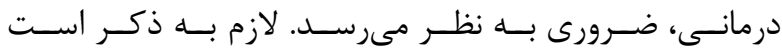

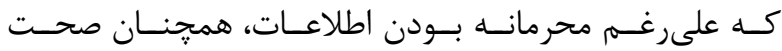

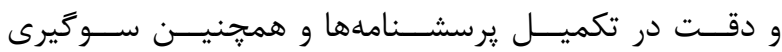

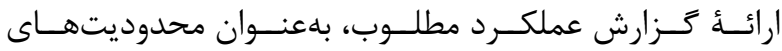

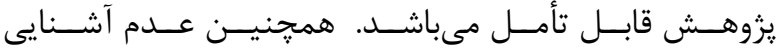

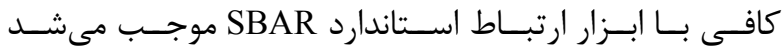

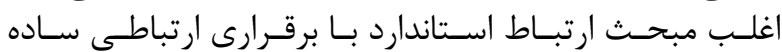

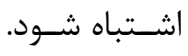

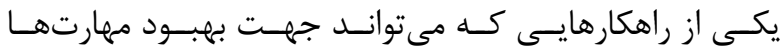

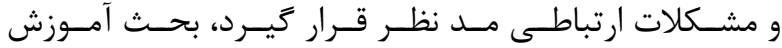

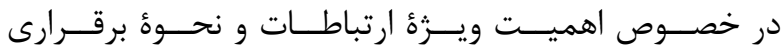

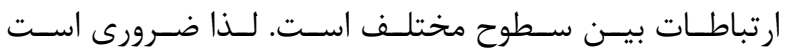

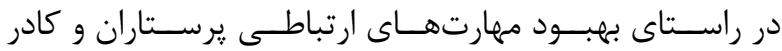

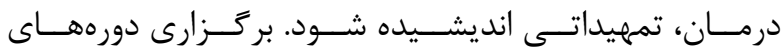

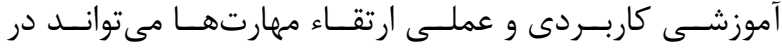

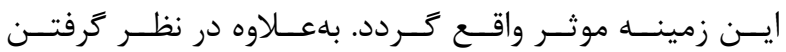

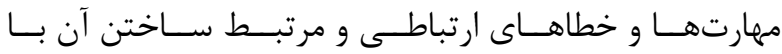

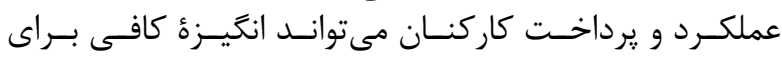

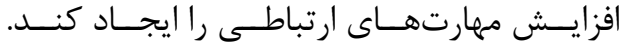

منابع

University of Medical Sciences. JHPM. 2012;1(1):45-54.

7. Brazpardnjany Sh, Shariati A, Alijani H, Moein Mousavi BS. Assessing barriers of Nurse-patient's effective communication in educational hospitals of Ahwaz. IJNR. 2010; 5(16): 45-52.

8. Kruijver IP, Kerkstra A, Bensing JM, van de Wiel HB. Nurse-patient communication in cancer care. A review of the literature. Cancer Nurs. 2000; 23(1): 20-31.

9. Zeighami Mohammadi S, Hagigi S. The relationship between the use of communication skills, communication and collaboration among nurses and doctors. Journal of Nursing and Midwifery. 2009; 6(4): 188-96.

10. Sweet S, Norman I. The nurse-doctor relationship: a selectiveliterature review. JAdv Nurs. 1995;22(1): 165-70.

11. Rezaei R, Hosseini SJ, Valaie N. Communication skills of doctors and their attitudes in Shiraz. J Kashan Univ Med Sci. 2001; 4(4): 19-26.

12. Dechairo-Marino A, Jordan-Marsh M, Traiger G, Saulo M. Nurse/physician collaboration: action research and the lessons learned. J Nurs Adm. 2001; 31(5): 223-32.

13. Van Ess Coeling H, Cukr PL. Communication styles 


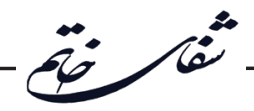

that promote perceptions of collaboration, quality, and nurse satisfaction. J Nurs Care Qual. 2000; 14(2): 63-74.

14. Awad SS, Fagan SP, Bellows C, Albo D, GreenRashad B, De la Garza M, et al. Bridging the communication gap in the operating room with medical team training. Am J Surg. 2005; 190(5): 770-4.

15. Elks KN, Riley RH. A survey of anaesthetists' perspectives of communication in the operating suite. Anaesth Intensive Care. 2009; 37: 108-11.

16. Kumar M, Har Dash H, Chawla R. Communication skills of anesthesiologists: an Indian perspective. J Anaesthesiol Clin Pharmacol. 2013; 29(3): 372-6.

17. Masroor d, Heidari Khayat N, Joolaei S. Review of patient safety events and its relation with professional interaction of physicians, nurses in nurses' perspectives. Journal of Nursing Management. 2012; 1(2): 35-47.

18. Rosenstein A, O'Daniel M. Impact and implications of disruptive behavior in the perioperative arena. J Am Coll Surg. 2006; 203(1): 96-105.

19. Zwarenstein M, Reeves S. Working together but apart: barriers and routes to nurse--physician collaboration. Jt Comm J Qual Improv. 2002; 28(5): 242-7.

20. Wheelan SA, Burchill CN, Tilin F. The link between teamwork and patients' outcomes in intensive care units. Am J Crit Care. 2003; 12(6): 527-34.

21. Zamani A, Zamani N, Sherafat Z. Evaluate and compare the attitudes of physicians and nurses (Alzahra hospital) about doctor and nurse communication cycle. J Isfahan Med School. 2010; 28(120): 1529-36.

22. Yousefi F. Emotional intelligence and communication skills of students. Journal of Iranian Psychologists. 2006; 3(9): 5-14.

23. Spitzberg BH. Methods of interpersonal skill assessment. Greene JO, Burleson BR. Handbook of communication and social interaction skills (Routledge Communication Series). 1st ed. Routledge. 2003.

24. Yukl G. How leaders influence organizational effectiveness. Leadersh Q. 2008; 19(6): 708-22.

25. Mathis R. Building bridges through effective communication. Supervision. 2007; 68(10(: 3-10.

26. Burke M, Boal J, Mitchell R. Communicating for better care: improving nursephysician communication. Am J Nurs. 2004; 104(12): 40-7.

27. Farhangi AA. Human relations. Tehran. Rasa cultural Institute. 2003.
28. Monajemizadeh A. The relationship between interpersonal communication skills and social acceptance of students, in Social Sciences. Master thesis. Isfahan, Islamic Azad University of Khorasgan Branch. 2012.

29. Victorian Government Department of Health. Promoting effective communication among healthcare professionals to improve patient safety and quality of care. Melbourne: Victorian Quality Council. 2010.

30. Thomas EJ, Sexton JB, Reich RLH. Discrepant attitudes about teamwork among critical care nurses and physicians. Crit Care Med. 2003; 31(3): 956-9.

31. Rostami H, Rahmani A, Ghahremanian A. Professional relationship between nurses, physicians in nurses' perspective. Journal of Nursing and Midwifery Gorgan, 2010; 7(1): 63-72.

32. Mullan BA, Kothe EJ. Evaluating a nursing communication skills training course: the relationships between self-rated ability, satisfaction, and actual performance. Nurse Educ Pract. 2010; 10(6): 374-8.

33. Ogbimi RI, Adebamowo CA. Questionnaire survey of working relationships between nurses and doctors in University Teaching Hospitals in Southern Nigeria. BMC Nursing. 2006; 5(2): 1-6.

34. Shafakhah M, Zarshenas L, SharifF, Sabet Sarvestani R. Evaluation of nursing students' communication abilities in clinical courses in hospitals. Glob J Health Sci. 2015; 7(4): 323-8.

35. Aghabarary M, Varvani A, Mohammadi E. Barriers to application of communicative skills by nurses in nursepatient interaction. Nurses and Patients' Perspective. 2009; 22(61): 19-31.

36. Karimi Moonaghi H, Taheri NK, Behnam Voshani $\mathrm{H}$, Vaghee S, Yavari M. The effect of communication skills training to nurses on the quality of patient care. Journal of Evidence Based Care. 2013; 2(5): 37-46.

37. Roter D, Rosenbaum J, de Negri B, Renaud D, DiPrete-Brown L, Hernandez O. The effects of a continuing medical education program in interpersonal communication skills on doctor practice and patient satisfaction in Trinidad and Tobago. Med Educ. 1998; 32(2): 181-9.

38. Snelgrove S, Hughes D. Interprofessional relations between doctors and nurses: perspective from south Wales. J Adv Nurs. 2000; 31(3): 661-7.

39. Rosenstein AH, Russell H, Lauve R. Disruptive physician behavior contributes to nursing shortage. Physician Exec. 2002; 28(6): 8-11. 